\title{
ANOTHER COUNTEREXAMPLE IN ANR THEORY
}

\author{
JAN VAN MILL
}

\begin{abstract}
We answer an old question due to Kuratowski by constructing a (separable metric) space $X$ having the following properties: (1) $X$ is not an ANR, and (2) for every space $Y$ and for every compact $A \subseteq Y$, every continuous map $f: A \rightarrow X$ can be continuously extended to a map $\bar{f}: Y \rightarrow X$.
\end{abstract}

1. Introduction. All spaces under discussion are separable metric, and for all undefined notions see [1, $\mathbf{3}$ and $\mathbf{5}]$.

A space $X$ has the compact extension property (abbreviated CEP) if for every space $Y$ and for every compact $A \subseteq Y$, every continuous function $f: A \rightarrow X$ can be extended to a continuous function $\bar{f}: Y \rightarrow X$. It is easy to show that $\mathrm{AR} \Rightarrow \mathrm{CEP} \Rightarrow C^{\infty}$ and $L C^{\infty}$. For details, see [3 and $\left.\mathbf{9}\right]$.

Clearly, for compact $X$ we have $X \in \mathrm{AR}$ if and only if $X \in \mathrm{CEP}$. In addition, if $X$ is finite-dimensional, then $X \in \mathrm{AR}$ if and only if $X \in C^{\infty}$ and $X \in L C^{\infty}$; see $[3,5]$. It is known that the property of being $C^{\infty}$ and $L C^{\infty}$ does not imply CEP since Borsuk [3] constructed an example of a contractible and locally contractible compactum which is not an AR. The question naturally arises whether CEP implies AR. This was asked by Kuratowski [9] in 1951. The aim of this note is to answer this question in the negative by constructing a space $X$ having the following properties:

(1) $X$ is not an ANR.

(2) For every space $Y$, for every analytic closed subspace $A \subseteq Y$, every continuous function $f: A \rightarrow Y$ can be extended to a continuous function $\bar{f}: Y \rightarrow X$.

Recall that a space is analytic if it is a continuous image of the space of irrational numbers. Clearly, every compact space is analytic. It is even true that every topologically complete space is analytic [10]. As in many counterexamples in ANR theory $[\mathbf{4}, \mathbf{1 1}]$, the Taylor example $[\mathbf{1 2}]$ is an essential ingredient in our example.

I am indebted to Tadeusz Dobrowolski for informing me about Kuratowski's question, to Doug Curtis for some useful information, and to R. D. Anderson for inviting me to visit Louisiana State University.

2. The example. By Taylor $[\mathbf{1 2}]$ there exists a compact space $T$ and a cell-like mapping $f: T \rightarrow M$, where $M$ is homeomorphic to the Hilbert cube $Q$, which is not a shape equivalence. We assume that $T$ is a $Z$-set in $Q$. Let $Z=Q \cup_{f} M$, and let $\pi: Q \rightarrow Z$ be the adjunction projection. It is well known that $Z$ is not an ANR since $\pi$ is not a hereditary shape equivalence. Clearly, $\pi$ is cell-like. Put $Y=Z \times I$, where $I$ denotes the interval $[0,1]$. Since $M \times I$ is compact, we can write it as the disjoint union of two sets, say $A$ and $B$, which both do not contain any Cantor set $[10$, p. 259]. Our example is

$$
X=(\pi(Q \backslash T) \times I) \cup A .
$$

Received by the editors May 6, 1985 .

1980 Mathematics Subject Classification. Primary 55M15.

Key words and phrases. Absolute retract, cell-like mapping, Taylor example. 
In the remaining part of this section we shall prove that $X$ is as required. $A R$.

2.1. LEMMA. If $F \subseteq A$ is countable, then $X(F)=(\pi(Q \backslash T) \times I) \cup F$ is an

ProOF. Define $\rho: Q \times I \rightarrow Y$ by $\rho=\pi \times \operatorname{id}_{I}$. Then $\rho$ is clearly cell-like. Put $S(F)=\rho^{-1}(X(F))$ and $\bar{\rho}=\rho \mid S(F)$. Then $\bar{\rho}: S(F) \rightarrow X(F)$ is cell-like. Since $(Q \backslash T) \times I \subseteq S(F)$ and $T \times I$ is a $Z$-set in the Hilbert cube $Q \times I$, the proof of [2, Theorem 3.1] shows that $S(F)$ is an AR. Consequently, $\bar{\rho}: S(F) \rightarrow X(F)$ is a cell-like mapping which is defined on an AR, while, moreover, its nondegeneracy set is contained in the countable subset $F$ of $X(F)$. This implies that $\bar{\rho}$ is a hereditary shape equivalence and in turn that $X(F)$ is an AR [8] (see also [1]).

2.2. LEMMA. For every space $E$ and every analytic closed $F \subseteq E$, every continuous function $f: F \rightarrow X$ can be extended to a continuous function $\bar{f}: E \rightarrow X$.

ProOF. Let $E$ be a space and let $F \subseteq E$ be closed and analytic. In addition, let $f: F \rightarrow X$ be continuous. Then $f(F)$ is analytic, and since $A$ is closed in $X$, $f(F) \cap A$ is closed in $f(F)$, whence $H=f(F) \cap A$ is analytic as well [10]. Since every uncountable analytic space contains a Cantor set [10] by the special choice of $A$ it follows that $H$ is countable. From Lemma 2.1 we therefore conclude that $f(F)$ is contained in the AR $X(H)$. Consequently, $f$ can be extended to a continuous function $\bar{f}: E \rightarrow X(H) \subseteq X$.

We shall now prove that $X$ is not an ANR.

\subsection{LEMMA. $X$ is not an $A N R$.}

Proof. To the contrary, assume that $X$ is an ANR. By Lemma 2.2 every continuous function $f: S^{n} \rightarrow X$, where $n \in\{0,1,2, \ldots\}$, extends to a continuous function $\bar{f}: B^{n+1} \rightarrow X$. Consequently, $X$ is $C^{\infty}$ and an ANR, so $X$ is in fact an AR [5, III, 7.3]. Let $N$ be a homeomorph of $Q$ containing $Y=Z \times I$. Define $T=N \backslash B$. Observe that $X$ is a closed subset of $T$. Since $X$ is an AR, there is a retraction $r: T \rightarrow X \subseteq Y$. Since $Y$ is compact, and hence topologically complete, there is a $G_{\delta}$-set $S$ of $N$ containing $T$ such that $r$ can be extended to a continuous function $\bar{r}: S \rightarrow Y$ (this is well known and easy to prove). Observe that $N \backslash S$ is an $F_{\sigma}$-subset of $N$ which is contained in $B$. Since $B$ contains no Cantor sets, it follows that $N \backslash S$ is countable. From [2, Theorem 3.1] we conclude that $S$ is an AR. Since $N \backslash S$ is countable, we find that $B \backslash S$ is countable, and, consequently, there exists a point $t \in I$ such that $(Z \times\{t\}) \cap(N \backslash S)=\varnothing$. Put $D=Z \times\{t\}$ and define $\xi: Y \rightarrow D$ by $\xi(x, s)=(x, t)$. Since $D \subseteq S$ and $r \mid(D \backslash B)$ is the identity, we also find that $\xi \circ \bar{r}$ is the identity on $D \backslash B$. Now since, clearly, $D \backslash B$ is dense in $D, \xi \circ \bar{r}$ is a retraction from $S$ onto $D$. Since, as was remarked above, $S$ is an AR and $D$, being homeomorphic to $Z$, is not, we have derived the desired contradiction.

Question. Let $X$ be an absolute Borel set with the compact extension property. Is $X$ an AR?

REMARK. A linear space $E$ is admissible if every compact subset of $E$ can be pushed by arbitrarily small maps into finite-dimensional linear subspaces of $E$. Every locally convex space is admissible, but there exist nonlocally convex spaces which are also admissible, e.g. $l^{p}$ for $p<1[\mathbf{7}]$. It is known that every admissible topologically complete linear space has the compact extension property [6]. Apparently, it is still unknown whether every linear space is admissible. 
ADDED IN PROOF. I recently showed that if there exists a cell-like dimension raising mapping between compact spaces then there exists a topologically complete non-ANR with the CEP.

\section{REFERENCES}

1. F. D. Ancel, The role of countable dimensionality in the theory of cell-like relations, Trans. Amer. Math. Soc. 287 (1985), 1-40.

2. R. D. Anderson, D. W. Curtis and J. van Mill, A fake topological Hilbert space, Trans. Amer. Math. Soc. 272 (1982), 311-321.

3. K. Borsuk, Theory of retracts, PWN, Warsaw, 1967.

4. R. J. Daverman and J. J. Walsh, Examples of cell-like maps that are not shape equivalences, Michigan Math. J. 30 (1983), 17-30.

5. S. T. Hu, Theory of retracts, Wayne State Univ. Press, Detroit, 1965.

6. V. Klee, Shrinkable neighborhoods in Hausdorff linear spaces, Math. Ann. 141 (1960), 281-285.

7. __ Leray-Schauder theory without local convexity, Math. Ann. 141 (1960), 286-296.

8. G. Kozlowski, Images of $A N R$ 's, unpublished manuscript.

9. K. Kuratowski, Sur quelques problèmes topologiques concernant le prolongement des functions continues, Colloq. Math. 2 (1951), 186-191.

10. K. Kuratowski and A. Mostowski, Set theory, PWN, Warsaw, 1976.

11. J. van Mill, A counterexample in ANR theory, Topology Appl. 12 (1981), 315-320.

12. J. L. Taylor, A counterexample in shape theory, Bull. Amer. Math. Soc. (N.S.) 81 (1975), $629-632$.

Subfaculteit Wiskunde en Informatica, VRIJe Universiteit, De BoelelaAN 1081, 1081 HV AMSTERDAM, THE NETHERLANDS

Mathematisch InstituUt, Universiteit VAN AMSTERdam, ROETERSSTRaAt 15, 1018 WB AMSTERDAM, THE NETHERLANDS 